\title{
Fatal Basilar Aneurysm Rupture 6 Months Following Pipeline Flow Diversion Treatment
}

\author{
Stylianos Pikis ${ }^{1}$ Tigran Petrosyan ${ }^{1} \quad$ Eftychios Archontakis² $\quad$ Georgios Arealis ${ }^{1}$
}

1Department of Neurosurgery, “Korgialenio Benakio" Red Cross Hospital of Athens, Athens, Greece

2Department of Interventional Neuroradiology, “Korgialenio Benakio" Red Cross Hospital of Athens, Athens, Greece

Indian J Neurosurg 2018;7:227-230

\begin{abstract}
Address for correspondence Stylianos Pikis, MD, MSc, Department of Neurosurgery, "Korgialenio Benakio" Red Cross Hospital of Athens, P.O. Box 11526, Athens, Greece (e-mail: steliospikis@hotmail.com).
\end{abstract}

\begin{abstract}
Keywords

- aneurysm

- rupture

- flow diverter

Background Very delayed aneurysmal rupture represents a rare, poorly understood, catastrophic complication of intracranial aneurysm flow diversion (FD) treatment.

Case Description A 48-year-old woman presented to the neurosurgical clinic for an elective admission 6-month post-FD treatment with a single pipeline embolization device (PED) treatment of a fusiform, large, midbasilar artery aneurysm. During her admission, the patient suffered a tonic-clonic seizure and collapsed. She was intubated and transferred for an urgent computed tomographic scan of the brain, which revealed subarachnoid hemorrhage and hydrocephalus. She was subsequently transferred to the operating room where an external ventricular drain was placed. Urgent diagnostic cerebral angiography revealed rupture of the previously treated aneurysm which was managed with deployment of a second PED and coil embolization of the right vertebral artery. Unfortunately, the patient succumbed to the disease 15 days later.

Conclusion The pathophysiologic mechanism responsible for delayed aneurysmal rupture post-FD treatment remains to be defined and may involve an acute rise in intra-aneurysmal pressures in a partially thrombosed aneurysm, continued hemodynamic stress on the aneurysmal wall due to persistent blood inflow, and thrombus-induced inflammation-mediated degradation the aneurysmal wall. Further clinical and anatomical studies are necessary to define the mechanisms responsible for delayed aneurysm ruptures and identify appropriate preventive measures.
\end{abstract}

\section{Introduction}

The introduction of flow diverters represents a major paradigm shift in the endovascular treatment of intracranial aneurysms from deconstructive, endosaccular, aneurysm treatment to reconstructive, endoluminal, diseased parent vessel treatment. The theoretical concept of flow diversion (FD) technology relies on the ability of flow diverters to induce a change in arterial and intra-aneurysmal hemodynamics leading to progressive thrombosis, occlusion, and subsequent healing of the aneurysm. ${ }^{1}$ However, the time course of the healing process to occur is unclear and is probably influenced by factors such as aneurysm size and morphology, the type of flow diverter used, the resultant flow change, the parent vessel geometry, and the patient's blood coagulation profile. ${ }^{12}$ Thus, an aneurysm may remain unprotected and prone to rupture for an uncertain period after endovascular FD treatment.

Delayed aneurysmal rupture post-FD treatment has been estimated to occur in $1 \%$ of all aneurysms treated and in $2 \%$ of aneurysms larger than $10 \mathrm{~mm} .{ }^{1}$ Very delayed aneurysm rupture occurring more than 3 months after FD has been rarely reported..$^{1-6}$ received

February 15, 2017

accepted

May 25, 2017

published online

October 12, 2017
DOI https://doi.org/

10.1055/s-0037-1607030. ISSN 2277-954X.
(C2018 Neurological Surgeons'

Society of India
License terms

(1) $\Theta \circledast$ 
We report on a rare case of a 48-year-old woman who suffered a fatal aneurysmal subarachnoid hemorrhage 6 months after FD treatment with a single pipeline embolization device (PED) of a large, fusiform, midbasilar artery aneurysm, and discuss the various mechanisms responsible for post-FD treatment delayed aneurysm rupture.

\section{Case Report}

A 48-year-old woman was referred to the neurosurgical outpatient clinic due to a large, fusiform, midbasilar artery aneurysm ( - Fig. 1A, B), diagnosed by magnetic resonance angiography of the brain during the investigation of a seizure. Neurologic evaluation was unremarkable. The patient reported being seizure free on Valproate. Informed consent for FD treatment was obtained. Following a 5-day treatment with clopidogrel $75 \mathrm{mg}$ and aspirin $100 \mathrm{mg} / \mathrm{d}$, the patient underwent deployment of a single PED (ev3, $4 \times$ $25 \mathrm{~mm}$ ) at the basilar artery (-Fig. 1C, D). Her postprocedural course was unremarkable, and she was discharged home on postoperative day 3 with instructions to continue her antiepileptic medication and the dual antiplatelet therapy until the 6 month digital subtraction angiography (DSA) follow-up.

The patient presented for cerebral DSA follow-up as scheduled. She reported being compliant with her medications and asymptomatic. During her admission, she had a tonic-clonic seizure and collapsed. She was intubated and transferred for computed tomographic scan of the brain, which demonstrated Fisher grade III subarachnoid hemorrhage (SAH) and hydrocephalus (-Fig. 2). An external ventricular drain was placed, and she was transferred to the angio suite. Cerebral DSA demonstrated partial thrombosis and residual filling of the aneurysm ( -Fig. 3A, B). A second PED was deployed in a telescoping fashion within the first followed by coil embolization of the right vertebral artery at the level of the right vertebral-basilar artery junction with substantial decrease in the aneurysmal sac blood flow noted (-Fig. $3 \mathbf{3 C}$ ). The patient was transferred to the intensive care unit for further management. However, she succumbed to the disease 15 days later.

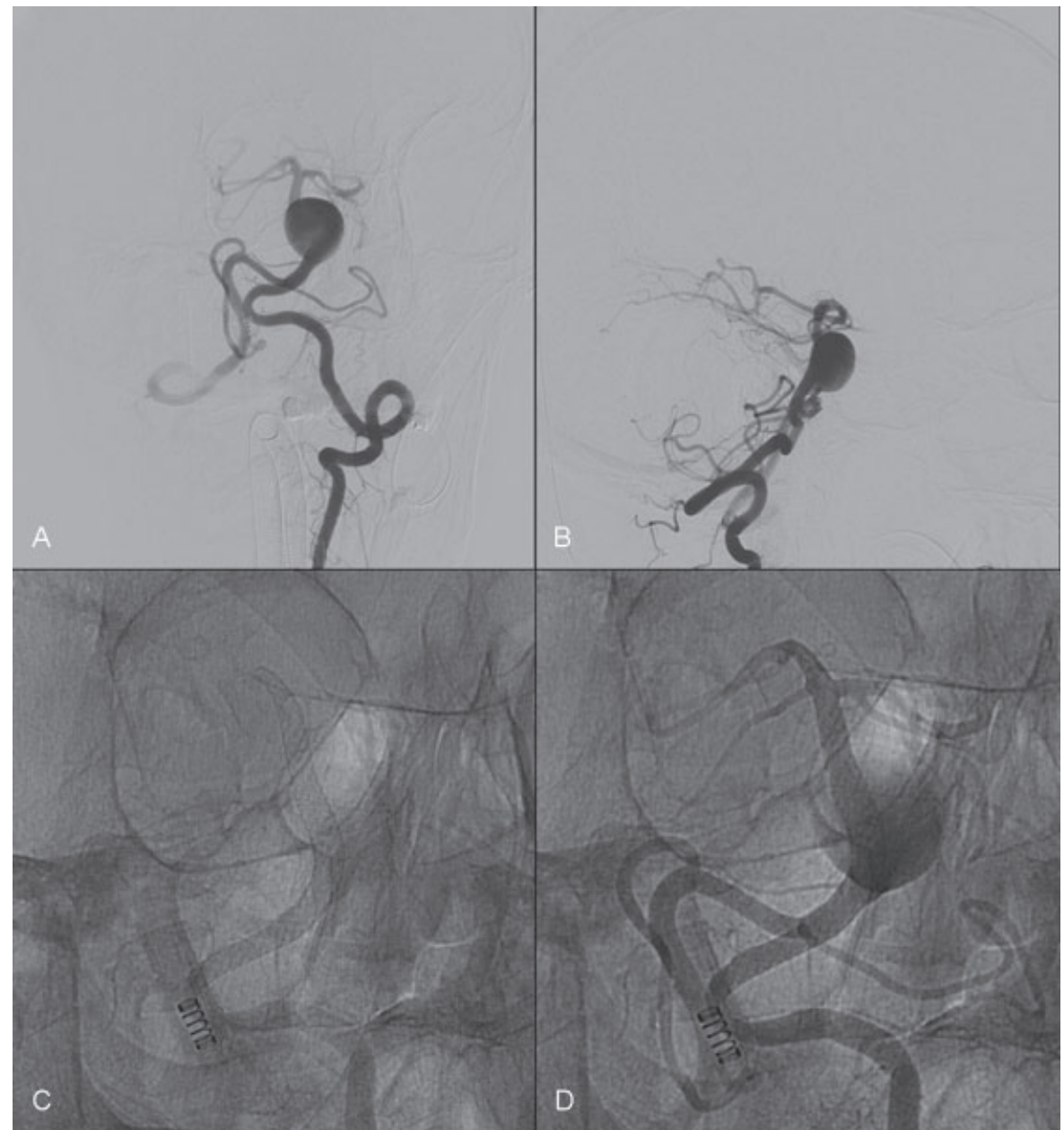

Fig. 1 (A) Anterior/posterior and (B) Lateral cerebral DSA demonstrating a large, saccular, midbasilar artery aneurysm. (C, D) Cerebral DSA posttreatment with a single pipeline embolization device $(4 \times 25 \mathrm{~mm})$. DSA, digital subtraction angiography. 


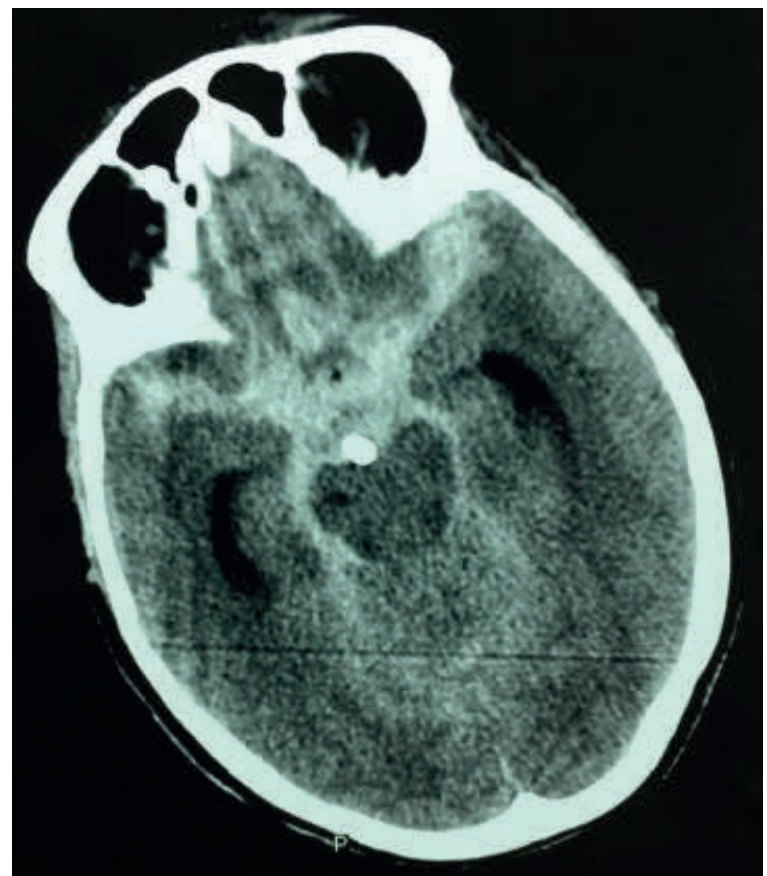

Fig. 2 Noncontrast computed tomographic scan of the head significant for Fisher grade III subarachnoid hemorrhage.

\section{Discussion}

Delayed aneurysmal rupture is a poorly understood, catastrophic complication of intracranial aneurysm FD-treatment. It has been estimated to occur with an incidence of $1 \%$ of all treated cases and with an incidence of $2.1 \%$ in aneurysms larger than $10 \mathrm{~mm} .{ }^{1}$ In a 2016 systemic review, $76.6 \%$ of delayed aneurysm ruptures occurred within 1 month of the procedure and resulted in death and poor outcome in $74.7 \%$ and in $6.7 \%$ of the cases, respectively. ${ }^{7}$ A higher risk of post-FD treatment delayed aneurysmal rupture has been suggested with (1) large and giant aneurysms, (2) symptomatic aneurysms, (3) saccular aneurysms with an aspect ratio (AR) of greater than 1.6, and (4) morphologic characteristics predisposing to an inertia-driven inflow. ${ }^{2}$

The mechanism responsible for delayed aneurysmal rupture after FD treatment is yet to be defined with two theories proposed: The first theory suggests that reduction in flow within the aneurysm following FD treatment corresponds to a rise in intra-aneurysmal pressures leading to rupture. ${ }^{89} \mathrm{Com}$ putational fluid dynamics (CFD) studies demonstrated that FD-induced intra-aneurysmal flow modification may lead to increases in intra-aneurysmal pressures ${ }^{9}$ and mural tension ${ }^{10}$ that especially in giant aneurysms, if high enough, may lead to rupture. ${ }^{9}$ This theory may explain the mechanism of early delayed aneurysmal rupture post-FD treatment. However, other CFD studies demonstrated no significant changes of intraaneurysmal pressures, indicating a minor role of pressure changes in delayed aneurysmal ruptures after FD. ${ }^{11,12}$ The second theory ${ }^{2,8,13}$ postulates that aneurysmal thrombosis may lead to an inflammatory reaction which depending on its severity may lead to normal healing, ${ }^{13}$ or if severe enough, to inflammation-mediated aneurysmal wall autolysis and rupture.,13

In our patient, the mechanism responsible for aneurysm rupture 6 months after FD treatment is unclear with five different scenarios possible: First, delayed aneurysm rupture occurred because of aneurysmal wall degradation due to the aggressive autolytic effects of the thrombus. Second, persistent hemodynamic stress on the wall of the aneurysm due to continued blood inflow subsequently led to rupture. Third, aneurysmal rupture occurred due to a complex interplay between FD-induced hemodynamic stress on the wall of the aneurysm and thrombosis-associated inflammation. Fourth, our patient suffered a witnessed seizure that may have been due to SAH. On the other hand, it may be that the seizure in our patient was unrelated to aneurysmal rupture. Seizure-associated rise in cerebral blood flow ${ }^{14}(\mathrm{CBF})$ and $\mathrm{CBF}$ velocities ${ }^{15}$ may have caused an acute rise in the hemodynamic stress along the wall of the partially thrombosed aneurysm leading to rupture. Fifth, partial thrombosis and residual filling of the aneurysm caused delayed aneurysmal rupture.

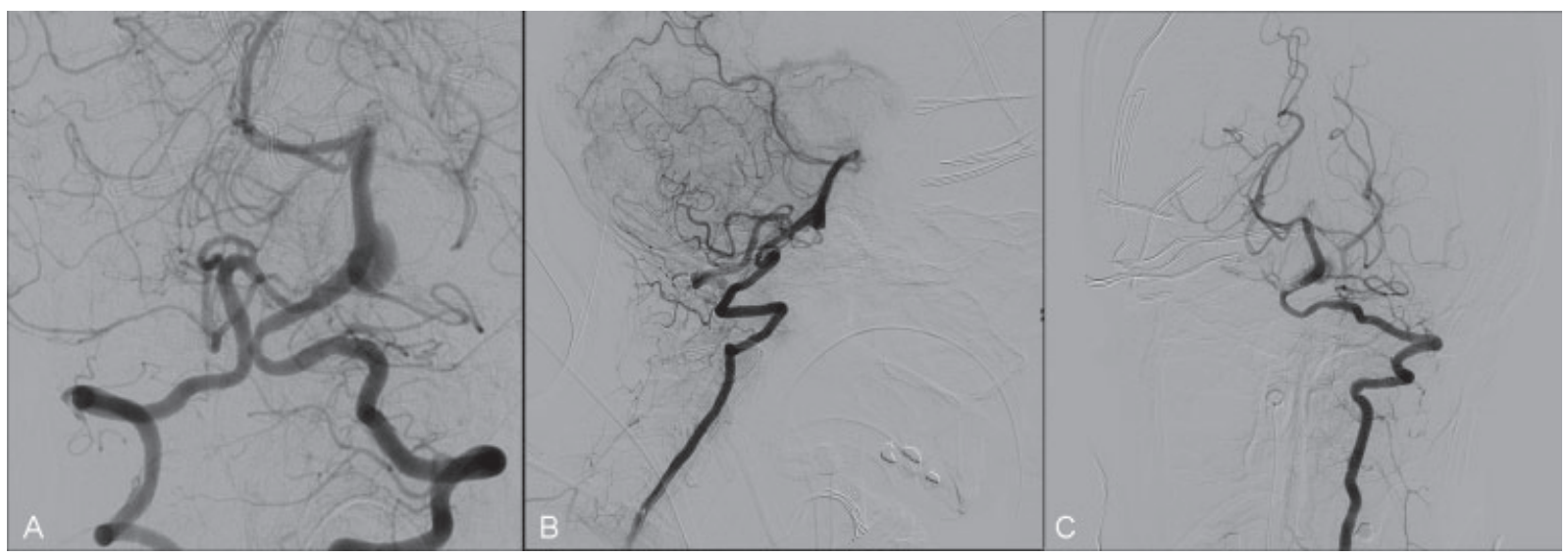

Fig. 3 (A) Anterior/posterior and (B) lateral cerebral DSA following aneurismal rupture and (C) cerebral DSA following deployment of a second pipeline embolization device and coil embolization of the right vertebral artery at the vertebrabasilar artery junction. DSA, digital subtraction angiography. 


\section{Conclusion}

In conclusion, we describe the rare case of a fatal basilar artery aneurysm rupture 6 months post-FD treatment. The pathophysiologic mechanism responsible for delayed aneurysmal rupture following FD treatment remains to be defined, and it may involve acute increases in intra-aneurysmal pressures in a partially thrombosed aneurysm, continued hemodynamic stress on the aneurysmal wall due to persistent blood inflow, and thrombus-induced inflammation-mediated degradation of the aneurysmal wall. Further clinical and anatomical studies are necessary to define the mechanisms responsible for delayed aneurysm ruptures and identify appropriate preventive measures.

\section{Funding}

None.

\section{Conflict of Interest}

None.

\section{References}

1 Kulcsár Z, Szikora I. The ESMINT Retrospective Analysis of Delayed Aneurysm Ruptures after flow diversion (RADAR) study. EJMINT Orig Artic. 2012;1244000078(October). http://www.ejmint.org/sites/default/files/pdf/original_article_1244000088.pdf. Accessed June 1, 2017

2 Kulcsár Z, Houdart E, Bonafé A, et al. Intra-aneurysmal thrombosis as a possible cause of delayed aneurysm rupture after flow-diversion treatment. AJNR Am J Neuroradiol 2011;32(1):20-25

3 Chalouhi N, Tjoumakaris S, Phillips JLH, et al. A single pipeline embolization device is sufficient for treatment of intracranial aneurysms. Am J Neuroradiol 2014;35(8):1562-1566

4 Saatci I, Yavuz K, Ozer C, Geyik S, Cekirge HS. Treatment of intracranial aneurysms using the pipeline flow-diverter embolization device: a single-center experience with long-term follow-up results. Am J Neuroradiol 2012;33(8):1436-1446
5 Monteith SJ, Tsimpas A, Dumont AS, et al. Endovascular treatment of fusiform cerebral aneurysms with the pipeline embolization device. J Neurosurg 2014;120(4):945-954

6 Berge J, Biondi A, Machi P, et al. Flow-diverter silk stent for the treatment of intracranial aneurysms: 1-year follow-up in a multicenter study. Am J Neuroradiol 2012;33(6):1150-1155

7 Rouchaud A, Brinjikji W, Lanzino G, Cloft HJ, Kadirvel R, Kallmes DF. Delayed hemorrhagic complications after flow diversion for intracranial aneurysms: a literature overview. Neuroradiology 2016;58(2):171-177

8 Briganti F, Leone G, Napoli M, Lauriola W, Florio F, Maiuri F. Early fatal hemorrhage after endovascular treatment of a giant aneurysm with flow diverter device and coils. Clin Neuroradiol 2015;25(2):201-205

9 Cebral JR, Mut F, Raschi M, et al. Aneurysm rupture following treatment with flow-diverting stents: computational hemodynamics analysis of treatment. Am J Neuroradiol 2011;32(1):27-33

10 Hassan T, Ahmed YM, Hassan AA. The adverse effects of flow-diverter stent-like devices on the flow pattern of saccular intracranial aneurysm models: computational fluid dynamics study. Acta Neurochir (Wien) 2011;153(8):1633-1640

11 Larrabide I, Aguilar ML, Morales HG, et al. Intra-aneurysmal pressure and flow changes induced by flow diverters: relation to aneurysm size and shape. Am J Neuroradiol 2013;34(4):816-822

12 Shobayashi Y, Tateshima S, Kakizaki R, Sudo R, Tanishita $\mathrm{K}$, Viñuela $\mathrm{F}$. Intra-aneurysmal hemodynamic alterations by a self-expandable intracranial stent and flow diversion stent: high intra-aneurysmal pressure remains regardless of flow velocity reduction. J Neurointerv Surg 2013;5 (Suppl 3):iii38-iii42

13 Berge J, Tourdias T, Moreau JF, Barreau X, Dousset V. Perianeurysmal brain inflammation after flow-diversion treatment. Am J Neuroradiol 2011;32(10):1930-1934

14 Monrad P, Sannagowdara K, Bozarth X, et al. Haemodynamic response associated with both ictal and interictal epileptiform activity using simultaneous video electroencephalography/ near infrared spectroscopy in a within-subject study. J Near Infrared Spectrosc 2015;23(4):209-218

15 Diehl B, Knecht S, Deppe M, Young C, Stodieck SRG. Cerebral hemodynamic response to generalized spike-wave discharges. Epilepsia 1998;39(12):1284-1289 